\title{
Pedestrians Egocentric Vision: Individual and Collective Analysis
}

\author{
Matteo Valsecchi \\ Dipartimento di Psicologia, Universitá di Bologna \\ Bologna, Italy \\ matteo.valsecchi@unibo.it \\ Raquel Gil-Rodriguez \\ Department of Psychology, Justus-Liebig University \\ Giessem, Germany \\ raquel.gil-rodriguez@psychol.uni-giessen.de
}

\author{
Arash Akbarinia \\ Department of Psychology, Justus-Liebig University \\ Giessem, Germany \\ arash.akbarinia@psychol.uni-giessen.de \\ Karl R. Gegenfurtner \\ Department of Psychology, Justus-Liebig University \\ Giessem, Germany \\ gegenfurtner@uni-giessen.de

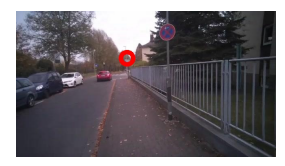 \\ Original RGB

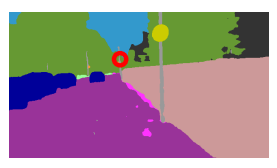 \\ Segmentation \\ [Zhu et al. 2019]

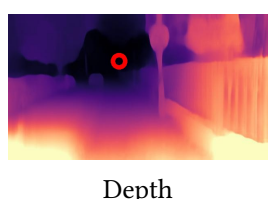 \\ Depth \\ [Godard et al. 2019]
} there substantial individual differences? To this end, we recorded egocentric video and gaze data from forty-three pedestrians. Here, we present this dataset designed to benchmark future research. The content of these videos was examined with respect to the depth and category of attended objects. We observe noticeable individual differences in both factors. Following these criteria, individual gaze patterns form a number of clusters. The unique signature of each set remains to be explored, whether it is based on low-level visual features or high-level cognitive characteristics.

\section{CCS CONCEPTS}

\section{- Applied computing $\rightarrow$ Psychology.}

\section{KEYWORDS}

Mobile Eye Tracking, Walking, Egocentric Video, Individual Differences, Database

\section{ACM Reference Format:}

Matteo Valsecchi, Arash Akbarinia, Raquel Gil-Rodriguez, and Karl R. Gegenfurtner. 2020. Pedestrians Egocentric Vision: Individual and Collective Analysis. In Symposium on Eye Tracking Research and Applications (ETRA '20 Short Papers), June 2-5, 2020, Stuttgart, Germany. ACM, New York, NY, USA, 5 pages. https://doi.org/10.1145/3379156.3391378

\section{INTRODUCTION}

Egocentric videos have become ubiquitous with the emergence of wearable cameras. This trend is expected to increase as these devices become smaller and more affordable [Betancourt et al. 2015] The fascination for recording the first-person perspective of our daily experience is ever-growing, and so is the interest in exploiting

Permission to make digital or hard copies of part or all of this work for personal or classroom use is granted without fee provided that copies are not made or distributed for profit or commercial advantage and that copies bear this notice and the full citation on the first page. Copyrights for third-party components of this work must be honored. For all other uses, contact the owner/author(s).

ETRA '20 Short Papers, June 2-5, 2020, Stuttgart, Germany

(C) 2020 Copyright held by the owner/author(s)

ACM ISBN 978-1-4503-7134-6/20/06.

https://doi.org/10.1145/3379156.3391378
Figure 1: An exemplary frame from the GEETUP dataset.

this source of data. Egocentric vision has proven itself as a promising technology in a wide range of applications [Bolanos et al. 2016], such as in health-related industries [Doherty et al. 2013] or navigation assistance to the visually impaired [Yagi et al. 2018]. Furthermore, egocentric videos offer a means to study human behaviour [Findlay et al. 2003]; specifically, when gaze data of camera wearer is also recorded.

Measuring eye movements in mobile settings has been technically possible for many years. However, its use was practically limited due to heavy headgear and bulky computers. This problem is partially solved when wearable eye trackers were released commercially [Bulling and Gellersen 2010]. The challenge now is to extract meaningful information from egocentric data. Manual annotation (e.g. fixated object, performed action, etc.) is only feasi[De Beugher et al. 2014; Parikh et al. 2019], researchers had to restrict the freedom of camera wearer (behaviour and action) and the explored environments [Hooge et al. 2019]. Recent advancements in computer vision offer an approach to automatically annotate video sequences. Consequently, a larger collection of egocentric videos in natural environments with unconstrained tasks can be analysed. We believe such large databases allow to benchmark different data analysis and modelling algorithms. We contribute to this line of investigation by:

(1) Introducing GEETUP (Giessen Egocentric Eye Tracking in Urban Paths), a dataset of egocentric videos accompanied with observers' gaze (see Figure 1). Due to the large number of participants ( 43 pedestrians), this dataset allows a systematic evaluation of individual and collective gaze patterns. The human gaze is denoted with the red circle. ble for a small number of videos. Thus, with a few exceptions, e.g. 
(2) Thoroughly analysing pedestrians' gaze with respect to two factors: the depth and category of attended objects. We observe clear distinctions among different individuals with regard to both factors. These variations are visualised and clustered using multidimensional scaling.

\subsection{Related works}

In one of the first attempts to collect a large eye-tracking data set, observers were limited to sitting on a chair while freely moving their head [Yamada et al. [n.d.]]. This dataset was never released. In another work, eye-tracking data was released without the accompanying egocentric videos [Steil and Bulling 2015]. Currently, GTEA Gaze [Fathi et al. 2012; Li et al. 2015] is the largest and most widely used dataset in the field. This is a collection of egocentric videos and recorded gaze data while observers cook specific recipes. The original research focus was on investigating the interaction of gaze and hand control while performing complex actions involving object manipulation. Nevertheless, those actions took place in a confined and stereotyped environment (kitchen). In our approach, observers perform the rather simple task of walking along an urban path, where interaction with objects is minimised. However, ego-motion is maximised and the environment is ever-changing, offering a wide range of possible fixation targets at different depths.

\section{METHODOLOGY}

\subsection{GEETUP Dataset}

The GEETUP (Giessen Egocentric Eye Tracking in Urban Paths) dataset is a collection of mobile eye-tracking data, collected as observers walked along two different urban paths (see Figure 2 for scene statistics ${ }^{1}$ ). The two routes (R1: $3.6 \mathrm{~km}$; R2: $3.2 \mathrm{~km}$ ) were largely independent, with little overlap that was in opposite directions. The observers were given a map with the route highlighted and instructed to follow it walking at their normal pace. They were asked to avoid using their smartphones during data acquisition. All recordings took place in autumn 2018, mostly under conditions of an overcast sky. In the case of a clear sky, observers were instructed to walk on the shady side of the street. A total of 43 observers $^{2}$ (all students) took part in the study. 39 walked on R1, 29 on R2, and 25 on both routes.

Recordings were done with two Tobii PRO glasses 2 eye trackers (Tobii Technology, Danderyd, Sweden). This device tracks gaze at $100 \mathrm{~Hz}$ and captures a head-fixed scene video of $1920 \times 1080$ pixels at $25 \mathrm{~Hz}$. Tobii Pro Glasses have been reported be stable in their calibration [Niehorster et al. 2019], and thus are suitable for prolonged measurements. After each walk, we informally checked this in its tracking accuracy. In total 59.9 hours have been recorded (33.1 hours on R1 and 26.8 hours on R2). On average observers spent 50.9 and 41.3 minutes on R1 and R2, respectively. We inspected the dataset to remove:

- indoor scenes as the observers left the building;

- poor quality videos due to sun glare or hair occlusion;

- episodes where observers looked at their smartphones, at the map, or at their hands;

\footnotetext{
${ }^{1}$ Note that only the percentages bigger than $2 \%$ are represented.

${ }^{2}$ Observers gave written consent approved by the ethical committee (LEK FB6 2017-08).
}

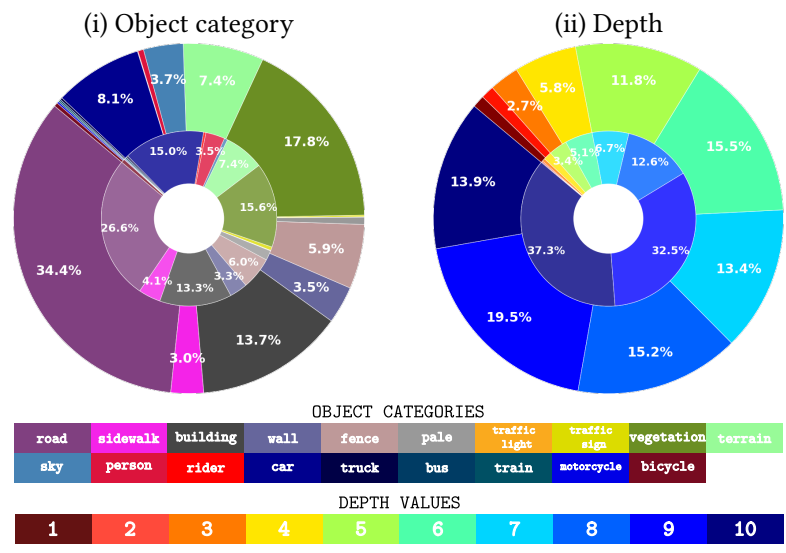

Figure 2: GEETUP overview. The frequency of objects' category and depth in the whole scene (outer circle) and at gaze (inner circle).

- episodes where observers were talking to people;

- episodes where observers were not walking, e.g. because they stopped in front of red lights or because they stopped for reading advertisements

- episodes where observers looked backwards while walking;

- episodes where the eye signal was missing for periods longer than 1 second.

Sections of the videos with a minimum duration of three seconds were then re-coded as the 5486 video segments that constitute the database (median duration 14.72 seconds, the maximum duration of 9.1 minutes). This reduced the total duration of the recording to 37.8 hours (20.5 hours for R1 and 17.3 hours for R2).

Segmenting the gaze data of a mobile eye-tracking into fixation and saccade events is difficult [Hessels et al. 2019]. This is even more challenging with constant head movements and locomotion that produce fast motion of gaze in the scene-camera frame of reference. We also observed a considerable amount of high temporal frequency noise, particularly in direct sunlight. For these reasons, we decided to compute the estimation of gaze position at each video frame as the median $X$ and $Y$ gaze position of the eye recording samples that were within a $25 \mathrm{~ms}$ timestamp of the video frame. This amounted roughly to 4 gaze samples per each video frame, i.e. the eye-tracking sampling rate is 4 times higher than the scene camera.

\subsection{Benchmarking}

GEETUP is suitable to benchmark egocentric vision. The content is diverse enough to capture the complexity of the real world. The number of participants is large enough to allow inter-individual comparisons. The amount of data is sufficient to allow for training models and testing their predictions within observers and across routes. For the benefit of the community, the entire dataset will be publicly released ${ }^{3}$. Below, we have discussed a few of features considered in this dataset.

Data Generate split sub-sets for: train (70\%), test (15\%) and validation (15\%). Sub-sets will be defined at three levels: individual

\footnotetext{
${ }^{3}$ http://www.allpsych.uni-giessen.de/GEETUP/index.htm
} 

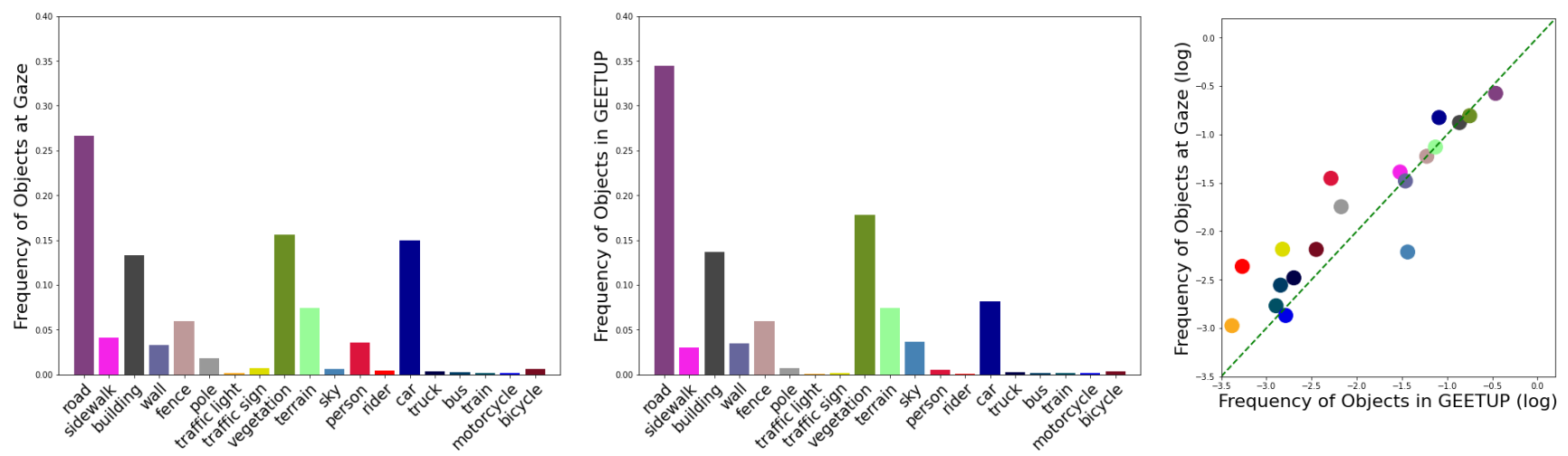

Figure 3: Left: the frequency of objects at gaze averaged over all participants. Middle: the frequency of objects in the entire scene (GEETUP) averaged over all participants. Right: the objects' frequency versus the objects' frequency at gaze.
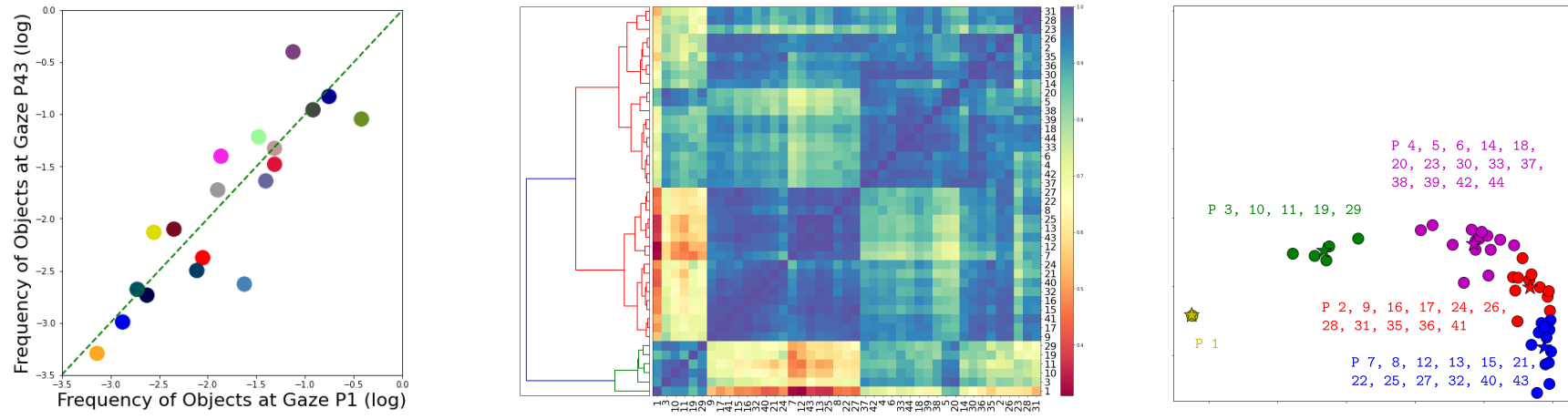

Figure 4: Individual comparison of objects at gaze. Left: an example from Participant 1 versus 43. Middle: hierarchical clustering of the Pearson coefficient between all the participants. Right: the MDS output on this matrix presented in 5 clusters.

participants, route-wise, and collectively for all. The ground truth of the test sets shall not be released and used to benchmark future models. The rationale is to allow for testing (a): whether a model of one individual pedestrian generalises well to everyone else; (b) what are the limitations of the collective model in predicting individual data; (c) the impact of the environment (i.e. type of route and appearance of uncontrolled entities) on gaze patterns.

Temporal challenges Different sequence lengths and frame rates can be combined to evaluate the impact of time integration in a given model. For example, (a) does a model trained on a particular frame rate generalise to other settings (i.e., due to camera specifications or lack of computational resources in embedded devices); (b) can a model produce faithful predictions in videos of any time duration accommodating actions that fade within a second or those lasting longer?

Multiple data modalities Along with the original RGB images, the segmentation map of objects [Zhu et al. 2019] and their depthmaps [Godard et al. 2019] are available for multi-modal fusion in a model. This is of interest from several perspectives. For instance, (a) how important is the depth of field in estimating of pedestrian gaze?; (b) are there intrinsic biases towards particular objects facilitating gaze prediction?; (c) what is the best strategy to conjoin high-level with low-level information?

\section{ANALYSING EGOCENTRIC VIDEOS}

The collected dataset contains the video frames alongside its corresponding gaze position (pixel coordinates). We analysed the data by investigating the relation of gaze with respect to depth and object category. To this end, we relied on state-of-the-art algorithms for object segmentation [Zhu et al. 2019], and depth estimation [Godard et al. 2019].

\subsection{Object categories}

The segmentation network in [Zhu et al. 2019] labels all pixels into 19 common objects present in city streets (road, sidewalk, person, car, vegetation, etc.). For each participant we computed the frequency of object categories (i) at gaze and (ii) in the whole scene; the left and middle panels in Figure 3, respectively. Note that both of them, in general terms, are correlated (right). The more frequent an object is in the sequence, the more likely it is to be at gaze, e.g. 'road' and 'vegetation' labels. There are exceptions to this observation: (a) the 'sky' category is highly present in the sequences, but participants hardly ever gaze at it, and (b) the 'person' and 'traffic lights' occur rarely in the sequences, but participants frequently gaze at them. This suggests that certain objects are more important to pedestrians while walking in an urban area. 

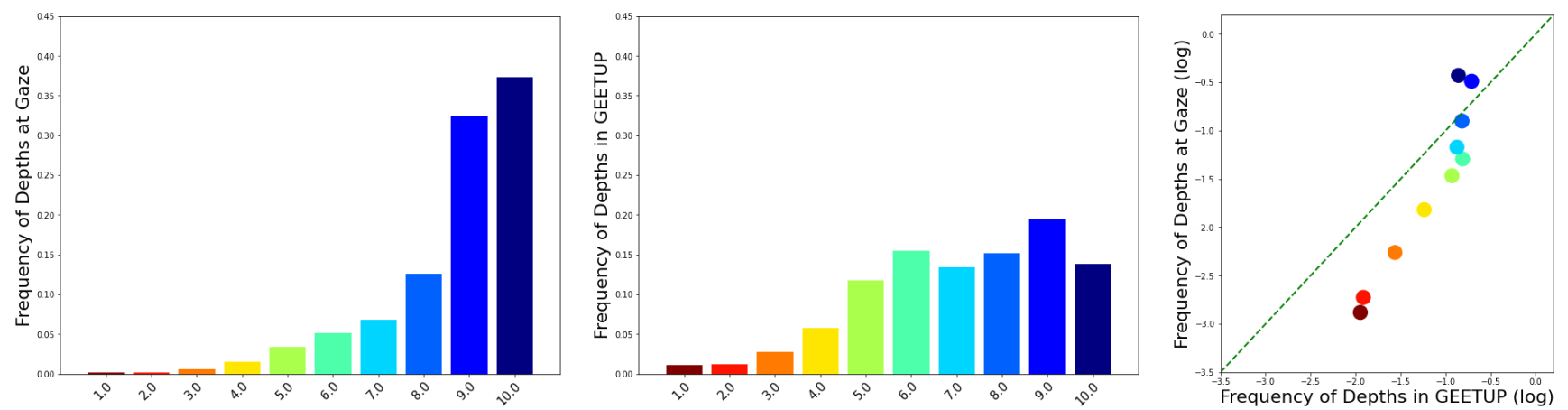

Figure 5: Left: the frequency of depths at gaze averaged over all participants. Middle: the frequency of depths in the entire scene (GEETUP) averaged over all participants. Right: the objects' frequency versus the depths' frequency at gaze.
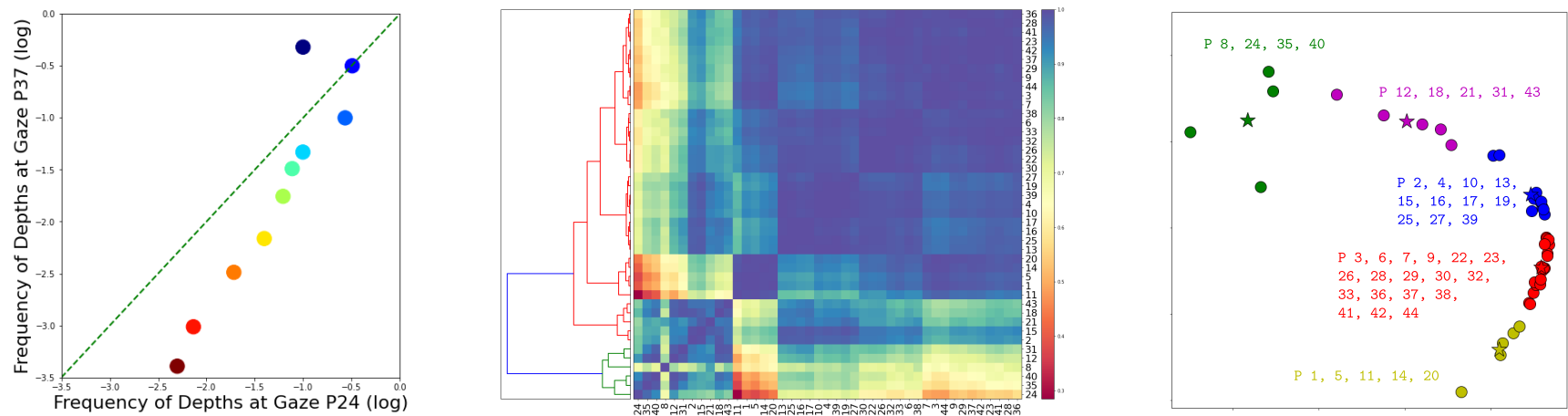

Figure 6: Individual comparison of attended depth. Left: an example from Participant 24 versus 37. Middle: hierarchical clustering of the Pearson coefficient between all the participants. Right: the MDS output on this matrix presented in 5 clusters.

The middle panel of Figure 4 compares the objects at gaze among all participants. This similarity is presented with a confusion matrix and dendrogram visualisation of hierarchical clustering [Jain and Dubes 1988]. Large individual differences can be observed (e.g. the participants 1 and 43 in the left panel). On the right, the multidimensional scaling representation (MDS) [Borg and Groenen 2006] is performed together with $\mathrm{k}$-means [MacQueen 1967] clustering $(k=5)^{4}$. The first participant is a cluster by herself since she focuses mostly on the 'vegetation' category. The green points belong to a group in which the predominant gaze is at 'vegetation', but other object categories like 'road' and 'car' are also relevant. In the set of magenta participants, the label 'road' is the most frequent object at gaze closely followed by the 'vegetation' and 'car' categories. Finally, the blue and red groups contain those participants that gazed mostly to 'road', with a large difference in the frequency of the rest of the categories. We are currently investigating whether these clusters coincide with previously reported individual differences in human gaze patterns [De Haas et al. 2019].

\subsection{Depth}

The network presented in [Godard et al. 2019] estimates the depthmap in the range of 0 to 10 (this is an arbitrary unit corresponding

\footnotetext{
${ }^{4}$ These two classification techniques use a distance matrix as an input. However, for visualisation purposes, we present the similarity matrix of Pearson coefficients.
}

to the relative depth of objects in the network's training set). For that reason, we decided to quantise them into 10 equidistant bins. Similar to the previous section, we computed the frequency of depth (i) at gaze and (ii) in the whole scene for each participant. Pedestrians tend to focus more on far-away objects (Figure 5, left), even though the distribution of depth values is rather uniform in the GEETUP dataset (the middle column). It should be highlighted that the correlation observed in the case of object categories (between an object at gaze and the entire scene) is reduced for the depth feature (Figure 5, right). Figure 6 presents the similarities between the pedestrians with respect to their depth at gaze and its dendrogram visualisation (middle) using hierarchical clustering. On the left, it is shown the scatter plot of the frequency of depth at gaze between subjects 24 and 37 . The right column corresponds to the MDS analysis of the similarity matrix by selecting 5 different clusters [MacQueen 1967]. For example, subject 24 belongs to the green cluster and subject 37 to the red one. The former cluster includes those pedestrians that look with higher frequency to near objects (a particular depth value is more frequent, but the rest is still relevant). The latter cluster corresponds to those pedestrians that exclusively focus on further distances and rather not attend closer objects. It is interesting to point out that the yellow cluster is an extreme case, where the frequency at gaze on very far objects represents more than $80 \%$ of the distribution, and most of the 
closer objects are almost negligible. For future work, we propose the object category and depth be analysed jointly in order to better understand individual differences.

\section{CONCLUSION}

In our data set of pedestrians egocentric vision-GEETUP-it is evident that patterns of human gaze are heterogeneous. Different individuals exhibit distinct habits. Some fixate roads more, while others pay attention to vegetation. Many look into the horizon and a few to closer objects. Reducing the dimensionality of these different gaze patterns with the multidimensional scaling (MDS) technique revealed a set of clusters. We are in the process of analysing characteristics of each group of individuals attempting to explain the root of these distinctions.

\section{ACKNOWLEDGMENTS}

This study was funded by Deutsche Forschungsgemeinschaft SFB/TRR 135 project $\mathrm{A} 8$.

\section{REFERENCES}

Alejandro Betancourt, Pietro Morerio, Carlo S Regazzoni, and Matthias Rauterberg. 2015. The evolution of first person vision methods: A survey. IEEE Transactions on Circuits and Systems for Video Technology 25, 5 (2015), 744-760.

Marc Bolanos, Mariella Dimiccoli, and Petia Radeva. 2016. Toward storytelling from visual lifelogging: An overview. IEEE Transactions on Human-Machine Systems 47, 1 (2016), 77-90.

Ingwer Borg and Patrick Groenen. 2006. Modern Multidimensional Scaling: Theory and Applications. Fournal of Educational Measurement 40 (06 2006), 277 - 280.

Andreas Bulling and Hans Gellersen. 2010. Toward mobile eye-based human-computer interaction. IEEE Pervasive Computing 9, 4 (2010), 8-12.

Stijn De Beugher, Geert Brône, and Toon Goedemé. 2014. Automatic analysis of in-thewild mobile eye-tracking experiments using object, face and person detection. In 2014 International Conference on Computer Vision Theory and Applications (VISAPP), Vol. 1. IEEE, 625-633.

Benjamin De Haas, Alexios L Iakovidis, D Samuel Schwarzkopf, and Karl R Gegenfurtner. 2019. Individual differences in visual salience vary along semantic dimensions. Proceedings of the National Academy of Sciences 116, 24 (2019), 11687-11692.

Aiden R Doherty, Steve E Hodges, Abby C King, Alan F Smeaton, Emma Berry, Chris JA Moulin, Siân Lindley, Paul Kelly, and Charlie Foster. 2013. Wearable cameras in health: the state of the art and future possibilities. American journal of preventive medicine 44, 3 (2013), 320-323.

Alireza Fathi, Yin Li, and James M Rehg. 2012. Learning to recognize daily actions using gaze. In European Conference on Computer Vision. Springer, 314-327.

John M Findlay, John M Findlay, Iain D Gilchrist, et al. 2003. Active vision: The psychology of looking and seeing. Number 37. Oxford University Press.

Clément Godard, Oisin Mac Aodha, Michael Firman, and Gabriel J Brostow. 2019 Digging into self-supervised monocular depth estimation. In Proceedings of the IEEE International Conference on Computer Vision. 3828-3838.

R.S. Hessels, D. C. Niehorster, M. Nystrom, R. Andersson, and I.T.C. Hooge. 2019. Is the eye-movement field confused about fixations and saccades? A survey among 124 researchers. (2019). European Conference on Eye Movements.

Ignace Hooge, Roy Hessels, Diederick Niehorster, Gabriel Diaz, Andrew Duchowski, and Jeff Pelz. 2019. From lab-based studies to eye-tracking in virtual and real worlds: conceptual and methodological problems and solutions. Fournal of Eye Movement Research 12, 7 (Nov. 2019). https://doi.org/10.16910/jemr.12.7.8

Anil K. Jain and Richard C. Dubes. 1988. Algorithms for Clustering Data. Prentice-Hall, Inc., USA.

Yin Li, Zhefan Ye, and James M Rehg. 2015. Delving into egocentric actions. In Proceedings of the IEEE Conference on Computer Vision and Pattern Recognition. 287-295.

J. MacQueen. 1967. Some methods for classification and analysis of multivariate observations. In Proceedings of the Fifth Berkeley Symposium on Mathematical Statistics and Probability, Volume 1: Statistics. University of California Press, Berkeley, California, 281-297.

D. C. Niehorster, T.C. Santini, R.S. Hessels, I.T.C. Hooge, E. Kasneci, and M. Nystrom 2019. When glasses slip: Data quality of mobile eye trackers during natural behavior. (2019). European Conference on Eye Movements.

D. Parikh, Y. Lu, Y. Xin, D. Wu, J. Pelz, and G. Lu. 2019. Where Am I Looking: Localizing Gaze In Reconstructed 3D Space. In 2019 IEEE Global Conference on Signal and Information Processing (GlobalSIP). 1-5.
Julian Steil and Andreas Bulling. 2015. Discovery of Everyday Human Activities From Long-Term Visual Behaviour Using Topic Models. In Proc. of the 2015 ACM International foint Conference on Pervasive and Ubiquitous Computing (UbiComp 2015) (2015-05-21). 75-85.

Takuma Yagi, Karttikeya Mangalam, Ryo Yonetani, and Yoichi Sato. 2018. Future person localization in first-person videos. In Proceedings of the IEEE Conference on Computer Vision and Pattern Recognition. 7593-7602.

Kentaro Yamada, Yusuke Sugano, Takahiro Okabe, Yoichi Sato, Akihiro Sugimoto, and Kazuo Hiraki. [n.d.]. Can saliency map models predict human egocentric visual attention?. In Asian Conference on Computer Vision (2010). Springer, 420-429.

Yi Zhu, Karan Sapra, Fitsum A Reda, Kevin J Shih, Shawn Newsam, Andrew Tao, and Bryan Catanzaro. 2019. Improving Semantic Segmentation via Video Propagation and Label Relaxation. In Proceedings of the IEEE Conference on Computer Vision and Pattern Recognition. 8856-8865. 